\title{
Relative capacities of time-gated versus continuous-wave imaging to localize tissue embedded vessels with increasing depth
}

\author{
Nimit L. Patel \\ Zi-Jing Lin \\ Yajuvendra Rathore \\ University of Texas at Arlington \\ Bioengineering Department \\ 501 West First Street \\ Arlington, Texas 76010
}

Edward H. Livingston

University of Texas Southwestern Medical Center at Dallas

Department of Surgery

5323 Harry Hines Boulevard

Dallas, Texas 75390-9156

Hanli Liu

George Alexandrakis

University of Texas at Arlington

Bioengineering Department

501 West First Street

Arlington, Texas 76010

\begin{abstract}
Surgeons often cannot see major vessels embedded in adipose tissue and inadvertently injure them. One such example occurs during surgical removal of the gallbladder, where injury of the nearby common bile duct leads to life-threatening complications. Nearinfrared imaging of the intraoperative field may help surgeons localize such critical tissue-embedded vessels. We have investigated how continuous-wave $(\mathrm{CW})$ imaging performs relative to time-gated widefield imaging, presently a rather costly technology, under broad Gaussian beam-illumination conditions. We have studied the simplified case of an isolated cylinder having bile-duct optical properties, embedded at different depths within a 2-cm slab of adipose tissue. Monte Carlo simulations were preformed for both reflectance and transillumination geometries. The relative performance of CW versus time-gated imaging was compared in terms of spatial resolution and contrast-to-background ratio in the resulting simulated images. It was found that time-gated imaging offers superior spatial resolution and vessel-detection sensitivity in most cases, though $\mathrm{CW}$ transillumination measurements may also offer satisfactory performance for this tissue geometry at lower cost. Experiments were performed in reflectance geometry to validate simulation results, and potential challenges in the translation of this technology to the clinic are discussed. ๑ 2010 Society of Photo-Optical Instrumentation Engineers. [DOI: 10.1117/1.3299728]
\end{abstract}

Keywords: diffuse reflectance; near infrared; time-resolved; ICCD; vessel imaging; bile duct.

Paper 09249PRR received Jun. 15, 2009; revised manuscript received Nov. 18, 2009; accepted for publication Dec. 1, 2009; published online Feb. 4, 2010.

\section{Introduction}

In recent years, the visualization of tissue embedded vessels at high spatial resolution during clinical procedures has been facilitated by several emerging technologies, such as photoacoustic imaging, ${ }^{1,2}$ photothermal radiometry, ${ }^{3}$ and optical coherence tomography. ${ }^{4}$ However, the tissue depth penetration of these methods is limited. For the investigation of deeper vessels by optical means, the multiple scattering of optical and near-infrared photons with tissues needs to be modeled appropriately. Spectrally resolved diffuse optical tomography has shown great potential for the localization and characterization of tissue-embedded absorbing heterogeneities. ${ }^{5-7}$ Heterogeneity localization is dependent on the spatial resolution of reconstructed images and characterization is dependent on the identification and quantification of their chromophore contents. However, the ill-posed nature of image reconstructions may compromise spatial resolution ${ }^{8}$ and chromophore content inference. ${ }^{9}$ These difficulties have spurred the development of some simplified, yet clinically powerful, approaches to either improve heterogeneity localization through image

Address all correspondence to: George Alexandrakis, University of Texas at Arlington, Bioengineering Department, 501 West First Street, Arlington, Texas 76010. Tel: 817-272-3496; Fax: 817-272-2251; E-mail: galex@uta.edu processing $^{10}$ or to improve characterization of tissueembedded chromophores through analysis of nontomographic measurements. ${ }^{11-15}$ When the tissues of interest can only be accessed from one side (e.g., the skin), depthwise heterogeneity localization and characterization becomes even more challenging. ${ }^{11,16}$ Fortunately, in some cases the clinician only needs to know where those heterogeneities are located and what the identity of their chromophore contents is, with no need to quantify the relative abundance of those chromophores. One such example arises when spectrally resolved reflectance measurements are performed to guide surgeons during a laparoscopic cholecystectomy procedure. ${ }^{17}$

Cholecystectomy is one of the most commonly performed operations in the United States, ${ }^{18}$ and its goal is the removal of the gallbladder. These operations are challenging because surgeons cannot directly visualize delicate structures adjacent to the gallbladder that are embedded in adipose tissue. Identification of these structures is particularly difficult when the cholecystectomy is performed laparoscopically, which is the primary approach used in the modern era. ${ }^{19}$ Laparoscopic procedures deprive the surgeon of tactile sensation, and by necessity, more emphasis is placed on image-guided resection of

$1083-3668 / 2010 / 15(1) / 016015 / 9 / \$ 25.00$ @ 2010 SPIE 
tissues. Standard laparoscopic equipment illuminates the operative field with light from a lamp that is delivered to the tissues of interest through a fiber-optic endoscope. The reflected image is projected onto a video screen. ${ }^{20}$ The biliary structures adjacent to the gallbladder cannot be visualized with standard equipment because they are embedded in adipose tissues contained within the gastrohepatic ligament. Turbidity resulting from the multiple scattering of light traveling through adipose tissue precludes direct visualization of biliary structures, causing the operating surgeon to guess at their location of based on prior experience. If that guess is inaccurate, then inadvertent injuries could be inflicted to these structures. These injuries are serious and lead to long-term morbidity and mortality. ${ }^{21}$ Patient safety during cholecystectomy would be significantly enhanced if there were imaging devices enabling surgeons to see through tissues and visualize various structures prior to, or during, dissection.

In this work, we evaluate the potential use of wide-field time-gated reflectance imaging as a means to improve the visualization of an adipose tissue embedded vessel having the optical properties of the bile duct. The main idea is that widefield continuous wave (CW) imaging, currently used in the clinic for laparoscopic procedures, ${ }^{17}$ produces images that carry mostly information about superficial tissue depths. It was hypothesized that time-gated imaging would enable collecting a larger proportion of photons that have traveled at greater tissue depths and thus help a surgeon visualize the bile duct when it is covered by several millimeters of adipose tissue. The concept of using time-gated reflectance to visualize absorbers embedded in deep tissues has been explored in some early optical mammography work. ${ }^{22-24}$ However, the tissue geometry and optical properties of the biliary tree environment warrant separate study because they are quite different compared to those of mammary tissues. In addition, this study explores the feasibility of detecting a vessel with increasing depth by use of wide-field intensified chargedcoupled device (ICCD) camera imaging. The latter is more applicable to the clinical setting because it enables higher speed of image formation relative to the spatial scanning of point detectors that earlier studies employed.

In this pilot study, we have compared the spatial resolution, quantified by the full width half maximum (FWHM) value, and the vessel-detection sensitivity, quantified by the contrast-to-background ratio $(\mathrm{C} / \mathrm{B})$, in reflectance images of the isolated bile duct embedded in adipose tissue. These quantitative comparisons were performed by analysis of timegated near-infrared reflectance images generated by Monte Carlo (MC) simulation. We simulated the reflectance detected by a time-gated camera, such as an ICCD, for a wide-field illumination geometry typically used in the clinic. Because the adipose tissue containing the biliary tree structures is relatively thin (i.e., $\sim 2 \mathrm{~cm}$ in thickness), we have also evaluated the relative potential of reflectance versus transillumination to visualize the bile duct at different adipose tissue depths. Simulation results were validated by performing time-gated wide-field reflectance measurements on tissue simulating phantoms with an ultrafast ICCD camera. Finally, the limitations in current experimental methods that need to be overcome for translating this methodology into the clinic are discussed.

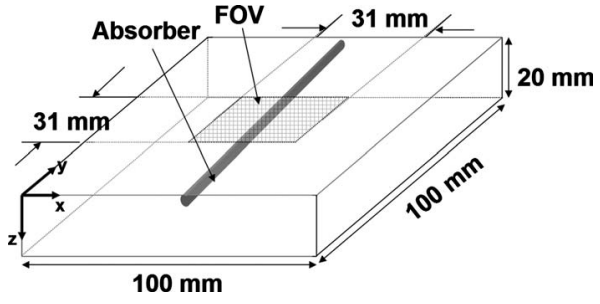

Fig. 1 Three-dimensional view of tissue geometry implemented for MC simulations.

\section{Methods}

\subsection{Simulations}

The tMCimg MC code $^{25}$ was run for simplified tissue geometries where the bile duct was assumed to be an infinite cylinder, of $4 \mathrm{~mm}$ diam, running parallel to the tissue surface. Simulations were performed for bile-duct depths ranging from 3 to $15 \mathrm{~mm}$ between the tissue surface and the vessel center. The optical properties of tissues, namely, the absorption coefficient $\mu_{a}$ and the transport scattering coefficient $\mu_{s}^{\prime}$, used to generate MC-simulated reflectance data, were collected from the literature. ${ }^{26-31}$ We verified that except for very early time gates, not relevant to this work, the MC data were identical for a typical tissue-scattering anisotropy ${ }^{32}$ of 0.9 versus isotropic scattering. The latter was therefore adopted, which decreased MC computation times substantially. Figure 1 shows the MC-simulation geometry. A $100 \times 100 \times 20 \mathrm{~mm}$ volume was assigned adipose tissue optical properties $\left(\mu_{a}=1.75\right.$ $\left.\times 10^{-3} \mathrm{~mm}^{-1}, \mu_{s}^{\prime}=1.10 \mathrm{~mm}^{-1}\right)$, and the bile duct was assigned the optical properties of human bile $\left(\mu_{a}=2.90\right.$ $\left.\times 10^{-1} \mathrm{~mm}^{-1}, \quad \mu_{s}^{\prime}=5.40 \times 10^{-1} \mathrm{~mm}^{-1}\right)$ for a near-infrared wavelength of $820 \mathrm{~nm}$. A $31 \times 31 \mathrm{~mm}$ array of $1 \times 1 \mathrm{~mm}$ detector pixels defined the simulated detector field of view (FOV).

Simulations were performed for transmission as well as reflectance mode using 150 million photons for every simulation case, which took $\sim 16 \mathrm{~h}$ to run on a $3-\mathrm{GHz}$ PC. The illumination profile was simulated as a $6-\mathrm{cm}$ FWHM Gaussian beam to match the one used in experiments. For the MC studies comparing reflectance to transmission data in the same tissue geometry, the Gaussian beam illumination was set vertical to the tissue surface. In reflectance experiments, the illumination angle was at $30 \mathrm{deg}$ to the surface normal so that the ICCD camera would not intersect the illumination path. In order to perform direct comparison to experiments, MC simulations were also run at a 30-deg illumination angle. All simulations were run for 15 contiguous time gates of 0.3 -ns width, each thus recording data up to a 4.5-ns delay, which was more than adequate for capturing the full time-resolved reflectance curves at all virtual detector pixel locations within the FOV. The instrument response function (IRF) of the ICCD setup was not simulated.

\subsection{Time-Gated Measurements with an ICCD Camera}

Figure 2(a) shows the block diagram of the ICCD timeresolved optical imaging system (LaVision $\mathrm{GmbH}$, Gottingen, Germany) employed for the phantom study. A picosecond pulsed laser (SC-450, Fianium Inc., Eugene, Oregon) pro- 

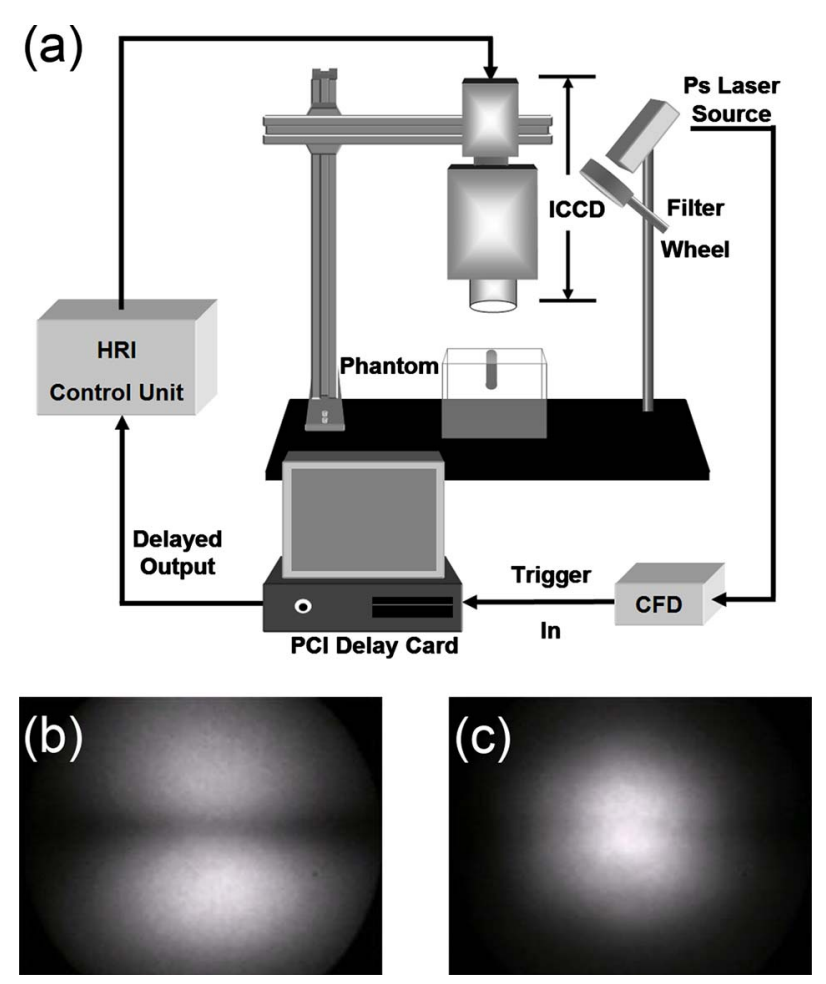

Fig. 2 (a) Block diagram of the ICCD imaging system and phantom illumination geometry. Examples of (b) time-resolved and (c) CW reflectance images of a capillary at 4-mm depth.

vided trigger pluses to a constant fraction discriminator that, in turn, triggered a high-rate imager (HRI) picosecond control unit (Kentech Instruments Limited, Oxfordshire, England) through a personal computer (PC) interface, adding adjustable time delays to the signal. The HRI control unit triggered ICCD image acquisition. The HRI gain was set to $400 \mathrm{~V}$, and 50 overlapping time gates of 0.3 -ns width were acquired with 0.05-ns step size, which resulted in a 2.5-ns scanned temporal range and a 320-ms integration time for each experiment. The ICCD camera unit consisted of a Cooke SensiCamQE CCD coupled to a gated image intensifier (PicoStar HR, LaVision). The picosecond laser emitted 450-1800 nm broadband pulses that passed through a 1064-nm short pass and an $820 \pm 10 \mathrm{~nm}$ filter (provided by LaVision) before reaching the tissue phantom surface. The laser pulse width was 5 ps and was operated at a pulse rate of $20 \mathrm{MHz}$ (one every $50 \mathrm{~ns}$ ). ICCD camera-specific software (Davis 7.0, LaVision) was used to control the image acquisition process. All measurements were taken in a dark room and saved on a PC. Before performing measurements on tissue phantoms, the system IRF was measured by reflecting the incident light beam onto a mirror rather than letting it propagate through the tissue phantom. The IRF was measured to be 300 ps at FWHM, which matched the system specifications.

The tissue-simulating phantom consisted of a tub filled with $1 \%$ Intralipid (Baxter Inc.), emulating the optical properties of adipose tissue in the semi-infinite medium geometry. Additionally, we performed some reflectance experiments using a porcine adipose tissue phantom to simulate somewhat more closely the clinical setting. The optical properties of the
$1 \%$ Intralipid $\left(\mu_{a}=3.40 \times 10^{-3} \mathrm{~mm}^{-1}, \quad \mu_{s}^{\prime}=0.92 \mathrm{~mm}^{-1}\right)$ and porcine adipose tissue $\left(\mu_{a}=1.80 \times 10^{-3} \mathrm{~mm}^{-1}\right.$, $\mu_{s}^{\prime}=1.00 \mathrm{~mm}^{-1}$ ) phantoms were determined at $830 \mathrm{~nm}$ by a frequency domain dual-channel oximeter (model no. 96208, ISS, Champaign, Illinois). For all phantom experiments, the bile duct was approximated by a 3-mm external diameter inkfilled capillary tube wrapped with black tape to augment its external diameter to $4 \mathrm{~mm}$. The depth of the capillary tube inside the phantom was controlled by a vernier scale, to which one of the capillary ends was attached. The tissue phantom surface was illuminated by a Gaussian profile beam of $6 \mathrm{~cm}$ FWHM, created by expanding the diameter of a $3.3-\mathrm{mm}$ Gaussian laser beam into a cone and illuminating from a distance of $\sim 1 \mathrm{~m}$ at a 30-deg angle [Fig. 2(a)]. In order to get the best possible focus, the ICCD camera was kept $25 \mathrm{~cm}$ above the surface of the intralipid phantom and was focused on its surface. The resulting FOV size was $38 \times 50 \mathrm{~mm}$ that was captured as a $1040 \times 1376$ pixel image. To avoid camera saturation, the tissue phantom illumination power was increased gradually during time-gated acquisition until the cumulative photon count reached a value of $\sim 3500$ at the time gate, having the maximum counts (maximum of 4096 counts for this 12-bit camera; 320-ms image capture time per time gate). To meet this requirement, illumination powers of 250-375 $\mathrm{mW}$ were used for time-resolved illumination, with higher powers being used for the larger capillary depths. To maintain the same image capture time during CW illumination the power was adjusted in the $150-200 \mathrm{~mW}$ range. The corresponding average illumination power densities across the detector FOV were $\sim 25$ and $\sim 14 \mathrm{~mW} / \mathrm{cm}^{2}$ for timeresolved and $\mathrm{CW}$ illumination, respectively. Consequently, $\sim 1.8$ times higher power was used for time-gated imaging relative to $\mathrm{CW}$ imaging.

In this setting, time-gated reflectance images were acquired for capillary depths of 2-8 $\mathrm{mm}$ in steps of $1 \mathrm{~mm}$ [e.g., Fig. 2(b)]. Images at depths $>8 \mathrm{~mm}$ were not acquired because the shadow of the capillary on the phantom surface was no longer detectable. Subsequently, a CW image was captured in dc mode [e.g., Fig. 2(c)] down to a maximum detection depth of $7 \mathrm{~mm}$. Comparison of Figs. 2(b) and 2(c) demonstrates how the capillary is less discernible in $\mathrm{CW}$ reflectance images than in time-resolved ones under Gaussian illumination. In order to make the reflectance profile across the detector FOV flatter and thus improve visibility of the capillary in reflectance images, we also performed control experiments with identical data acquisition settings, but in the absence of the capillary. The reflectance images in the presence and absence of the capillary were then divided, and the $\mathrm{C} / \mathrm{B}$ and FWHM values were quantified for all time gates by the processing algorithm described in Section 2.3. Subsequently, the single time gate having the highest $\mathrm{C} / \mathrm{B}$ and the one with the smallest FWHM were identified as the optimal ones for each metric, respectively. It is these latter values that were quantified in this work as a function of capillary depth to assess the ICCD system's vessel localization capacity.

Integration of data from several adjacent time gates did not offer any advantage in further improving the optimal $\mathrm{C} / \mathrm{B}$ and FWHM values. More specifically, for capillary depths of $<10 \mathrm{~mm}$, sufficient photons were detected per time gate and, therefore, averaging could not offer any significant improve- 
ment in photon statistics. For capillary depths beyond $10 \mathrm{~mm}$, the illumination laser power could not be further increased so that more photon counts per time gate could be collected and averaging of the optimal time gates with neighboring, nonoptimal, ones did not yield any substantial improvement in $\mathrm{C} / \mathrm{B}$ and FWHM values. We also investigated whether these two image-derived parameters could be further optimized by deconvolving the system IRF. We found that deconvolving the latter did not have a significant effect on the optimal C/B and FWHM values. Possible reasons for that were the small IRF width relative to the temporal extent of reflectance curves and the fact that all data analyzed in this work were a ratio between two measurements - one with and one without the capillary inside the tissue phantom.

\subsection{Data Processing Algorithm}

The ratio images were fitted by a nonlinear least-squares algorithm (fit.m, Matlab) to a Lorentzian curve shown below

$$
f(x)=a-\left[\left(\frac{b}{2 \pi}\right)\left(\frac{d}{(x-c)^{2}+(d / 2)^{2}}\right)\right],
$$

where $a$ represents the Background intensity level, $c$ represents the center, $d$ affects the width and amplitude, and $b$ is the amplitude of the Lorentzian reflectance shadow cast by the capillary on the phantom surface. The confidence interval for each of these four fitted parameters was computed using the "confint" function in Matlab. Figure 3(a) explains the quantities defining FWHM, C/B, which were used to compare the performance of $\mathrm{CW}$ versus time-gated imaging. The contrast was defined as the height of the Lorentzian, given by

$$
\text { contrast }=\left(\frac{2 b}{\pi d}\right),
$$

while the value of $d$ represents the FWHM of the fitted curve. The uncertainty in $\mathrm{C} / \mathrm{B}$ was computed by appropriate error propagation of the involved fitting parameters. It was empirically found that, for the MC-simulated and experimental images analyzed in this work, $\mathrm{C} / \mathrm{B}$ values less than $\sim 10 \%$ resulted in fits that did not converge.

\section{Results}

\subsection{Simulations}

The MC-simulated images for all time gates were fit to Eq. (1) for all bile-duct depths. Figures 3(b) and 3(c) demonstrate sample fits of time-gated reflectance profiles to the Lorentzian curve. It is seen that at later time gates, contrast decreases and FWHM increases. Early time gates had low contrast values because photons had primarily traveled above the absorber; thus, there was only a weak signature of the absorber in the reflectance data. Therefore, we investigated the question of which time gates offered optimal C/B and FWHM values and how much better these values were compared to the ones obtained by $\mathrm{CW}$ imaging.

The overall pattern observed was that contrast was initially low, then increased at later time gates, and then decreased again at later times. However, as discussed further later in Fig. $7(\mathrm{a})$, in the comparison with experimental data, C/B grew from a low value to its maximum and then stayed relatively
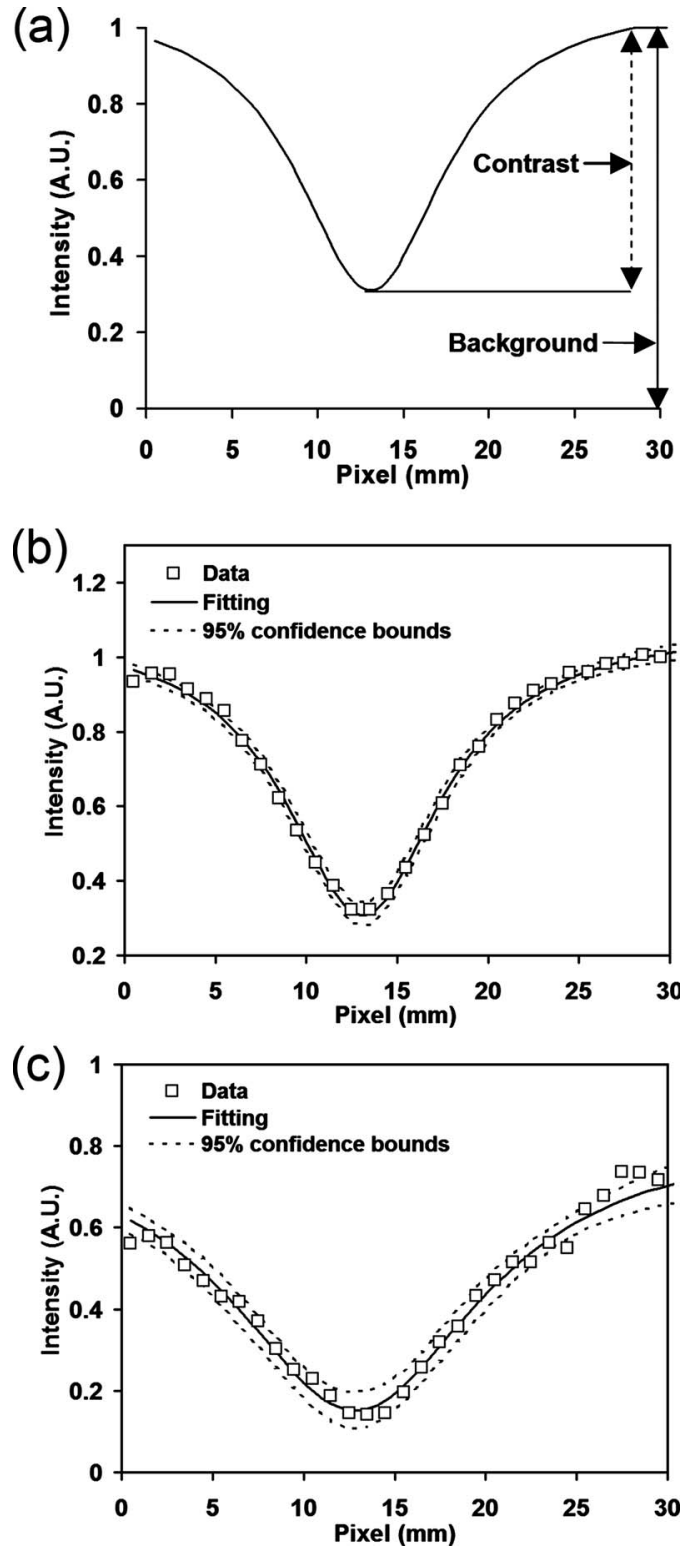

Fig. 3 (a) Schematic showing fitted parameters for a Lorentzian. Example of fits to a Lorentzian profile at (b) early and (c) late (210 ps and $1.47 \mathrm{~ns}$, respectively) time-gated reflectance images for a capillary at $5-\mathrm{mm}$ depth.

constant for later time gates. The reason was that background was decreasing along with contrast at the same rate, so that their ratio remained constant at a plateau value at all later time gates. We have selected the $\mathrm{C} / \mathrm{B}$ value attained at the earliest time gate for which that plateau was reached as the optimal parameter choice for vessel detection sensitivity. The reason for selecting the earliest plateau time gate was that fewer photons were detected at subsequent time gates. As a result, later time gates had inferior photon statistics and therefore had higher uncertainty in $\mathrm{C} / \mathrm{B}$ values, as seen later in Fig. 7(a). Figure 4(a) shows the resulting plots when comparing maximum $\mathrm{C} / \mathrm{B}$ values as a function of bile-duct depth for reflectance versus transmission mode. It is seen that the two illumination modes are almost equivalent down to a depth of $10 \mathrm{~mm}$, but the performance of transmission mode at greater 

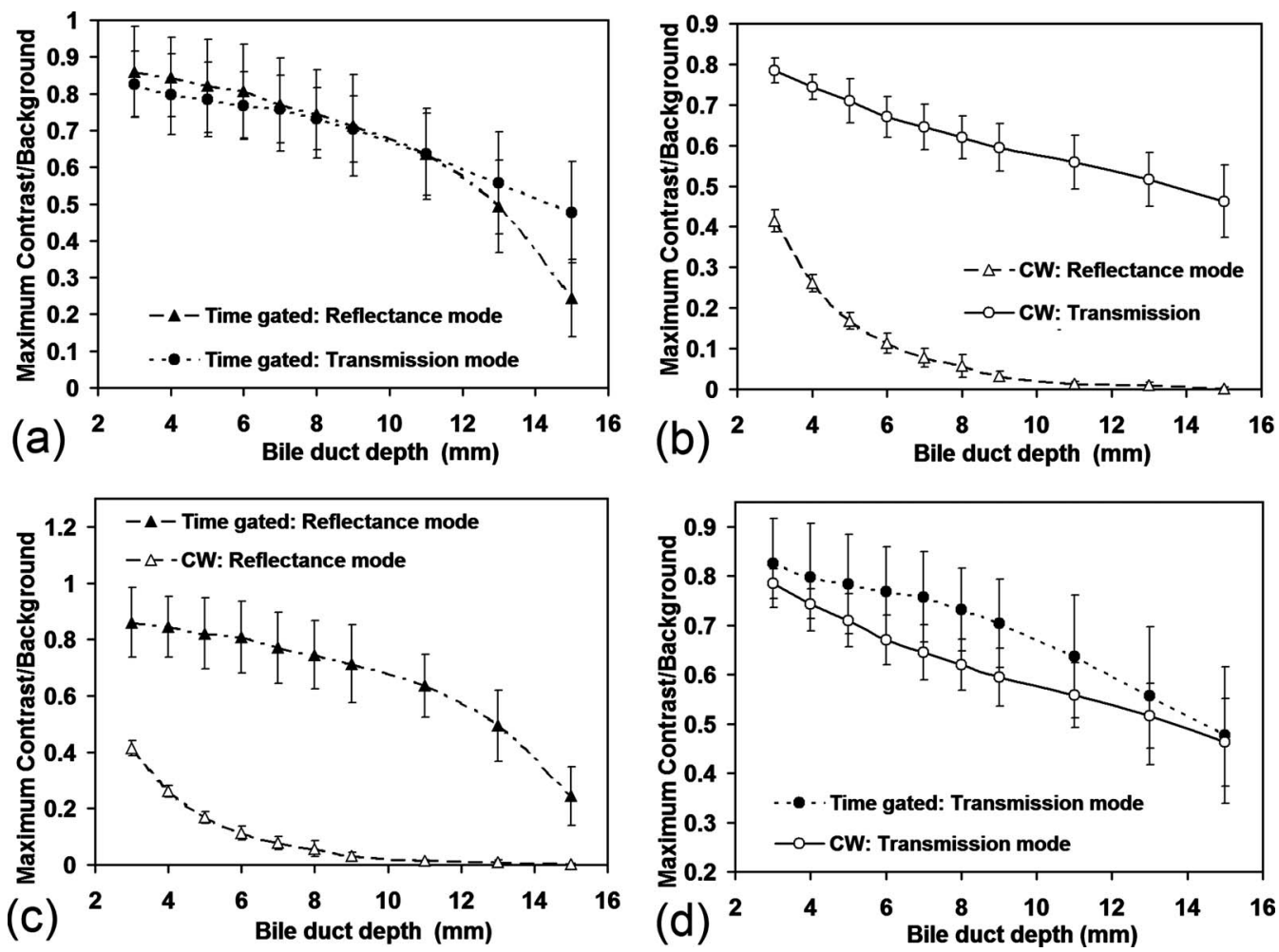

Fig. 4 Maximum C/B values attained as a function of bile-duct depth in (a) time-gated reflectance versus transmission mode, (b) CW reflectance versus transmission mode, (c) time-gated versus CW reflectance mode, and (d) time-gated versus CW transmission mode.

depths is superior. This is because more photons travel through the bile duct and therefore cast a greater absorption signature at the detectors, when this vessel is located closer to the transilluminating light source at the bottom of the 2-cm-thick adipose tissue slab. Therefore, the transmission time-gated mode appears to be the preferable illumination geometry for attaining good contrast for all bile-duct depths, even though the time-gated reflectance mode may have a marginal advantage at superficial depths. The advantage of transmission mode is far superior for $\mathrm{CW}$ illumination as can be seen in Fig. 4(b). In this case the early photons, which travel only superficially and above the bile duct, are the main contribution to the reflectance signal, which dramatically reduces the $\mathrm{C} / \mathrm{B}$ ratio.

The capacity of time-gated images for depth selectivity enables a substantial improvement of C/B (i.e., improved detection sensitivity) over CW, as is clearly seen in Fig. 4(c). However, the depth selectivity of time-gated imaging is reduced when imaging in reflectance mode for greater bile duct depths. This is because the photons that have traveled through the bile duct and make it back to the surface must have scattered many times, and due to the diffusive nature of this process, they could have visited a wide range of tissue depths. Therefore, in Fig. 4(c) it is seen that C/B values for timegated imaging converge toward those of $\mathrm{CW}$ imaging for greater bile-duct depths. Interestingly, the detection sensitivity in $\mathrm{CW}$ transmission mode is only slightly inferior (i.e., by no more than $\sim 20 \%$ ) to the one for time-gated transmission imaging [Fig. 4(d)]. This is because in transmission-mode photons can travel through the bile duct before reaching the detectors on the other side of the adipose tissue slab. We conclude that $\mathrm{CW}$ imaging in transmission mode may in fact be the simplest means of attaining good detection sensitivity of the bile duct, though time-gated imaging is superior in most cases.

The superiority of transmission relative to reflectance imaging was also confirmed by the improved spatial resolution, as quantified by the minimum FWHM values attained in the time-gated images. Figure 5(a) demonstrates that time-gated transmission attains better spatial resolution than time-gated reflectance as bile-duct depth increases, with FWHM improving by a factor of 2 at a depth of $15 \mathrm{~cm}$. In reflectance mode, fewer photons reach the bile duct when it is located deeper in tissue. As a result the wide-field reflectance images become very noisy, which has increased the uncertainty of fitted FWHM values. Similarly, Fig. 5(b) demonstrates that transillumination also yields superior spatial resolution for the $\mathrm{CW}$ mode, especially at greater bile-duct depths. However, the spatial resolution of time-resolved illumination is not superior to $\mathrm{CW}$ illumination in reflectance mode but is only superior in transmission mode as demonstrated in Figs. 5(c) and 5(d), respectively. This is due to the weak depth selectivity that wide-field time-gated imaging can attain relative to the complete lack thereof in $\mathrm{CW}$ imaging.

Simulations for bile-duct depths of $>15 \mathrm{~mm}$ are not shown in Figs. 5 and 6 because the shadow of the bile-duct cast at the detectors was very shallow and therefore overwhelmed by MC stochastic noise. To improve the scoring statistics and remedy this problem, MC simulations would need to be run for substantially larger numbers of photon histories. Therefore, the plotted error bars that originated from the fitted parameter standard deviations could have been 

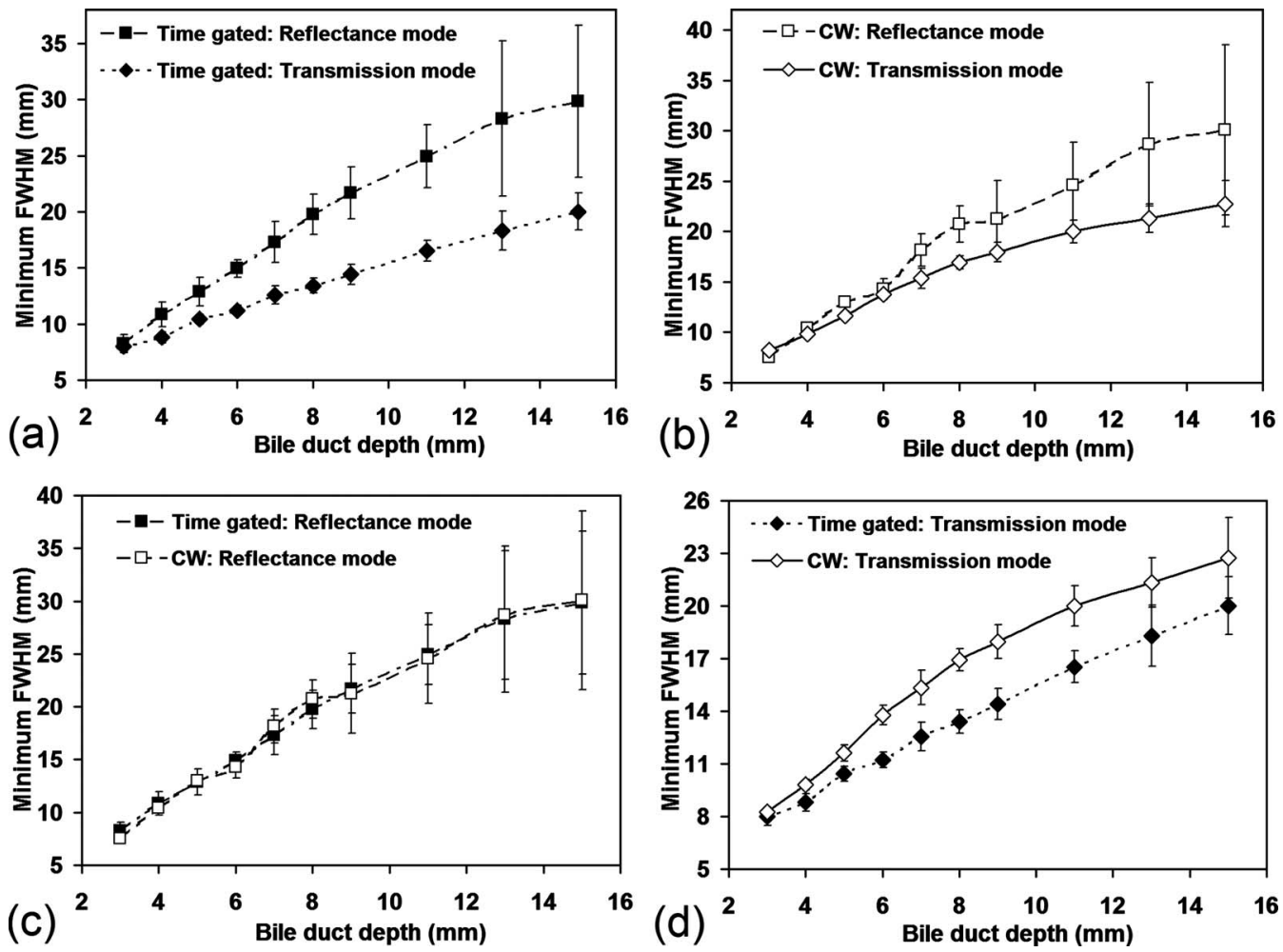

Fig. 5 Minimum FWHM values attained as a function of bile duct depth in (a) time-gated reflectance versus transmission mode, (b) CW reflectance versus transmission mode, (c) time-gated versus CW reflectance mode, and (d) time-gated versus CW transmission mode.

smaller magnitudes if longer MC runs were performed. However, the computational times involved would be impractical. This is particularly true for the time-resolved simulations, which spread the same amount of simulated photon histories over a large number of time gates. It is also important to mention that fewer photons would be scored at detectors closer to the edge of the Gaussian illumination profile, which could further reduce the maximum depth at which a vessel could be detected. In this work, the linear dimension of the detector array $(3.1 \mathrm{~cm})$ was substantially smaller than the illumination profile's FWHM $(6 \mathrm{~cm})$, which resulted in negligible variation of optimal C/B and FWHM values, and therefore, in depth detection sensitivity, across the FOV.

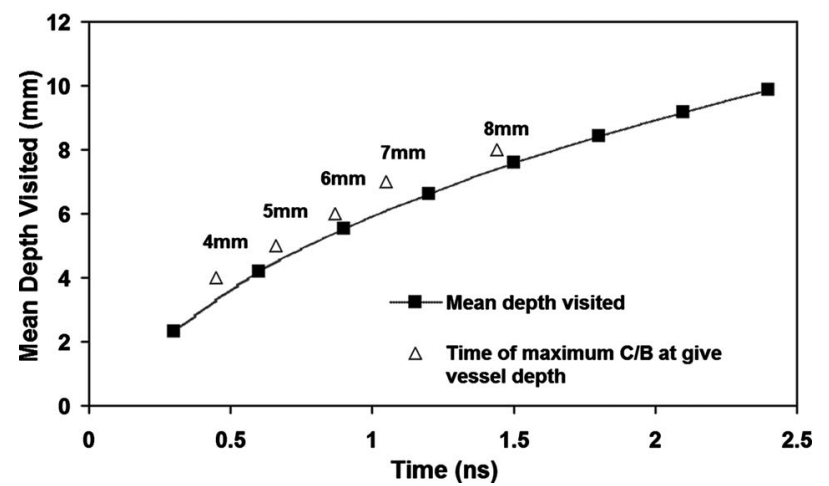

Fig. 6 Maximum C/B for a given vessel depth was attained at a time delay nearly equal to the time that incident light took to reach a mean visit tissue depth equal to the vessel's center depth.
One final consideration for Figs. 4 and 5 was whether there was any relation between the time gates at which maximum $\mathrm{C} / \mathrm{B}$ versus a minimum FWHM was attained. As the tMCimg ${ }^{25}$ code can score the photon weights as a function of time gate and depth in tissue, we have computed the mean depth visited as a function of time delay. Figure 6 plots this latter quantity as well as the time delays at which maximum $\mathrm{C} / \mathrm{B}$ was attained for a given vessel depth. It is seen that maximum $\mathrm{C} / \mathrm{B}$ was attained when the mean visit depth was nearly equal to the depth of the vessel's center. On the other hand, minimum FWHM was attained at the earliest possible time gate where the shadow of the tissue-embedded vessel could be seen in the reflectance profile, corresponding to tissue depths akin to the top side of the vessel (not shown in Fig. 6), and reliably fitted to Eq. (1).

\subsection{Comparison between MC Simulations and ICCD Camera Experiments}

We have also performed time-gated reflectance experiments on tissue-simulating phantoms, mimicking the MC-simulation geometry, with an ICCD camera as described in Section 2. Figure 7(a) shows that the time-gated $\mathrm{C} / \mathrm{B}$ values for a 4-mm-diam ink-filled capillary, mimicking the bile duct at $4 \mathrm{~mm}$ depth, agree rather well with simulation data. Both the ink and the bile-duct absorption coefficient assumed in the simulations ${ }^{29}$ have very high values; thus, exact matching in their absorption values was not necessary. Similar agreement in $\mathrm{C} / \mathrm{B}$ versus time delay was attained for greater vessel depths. However, acquisition of such $\mathrm{C} / \mathrm{B}$ versus time-delay curves proved very challenging due to the limited dynamic 

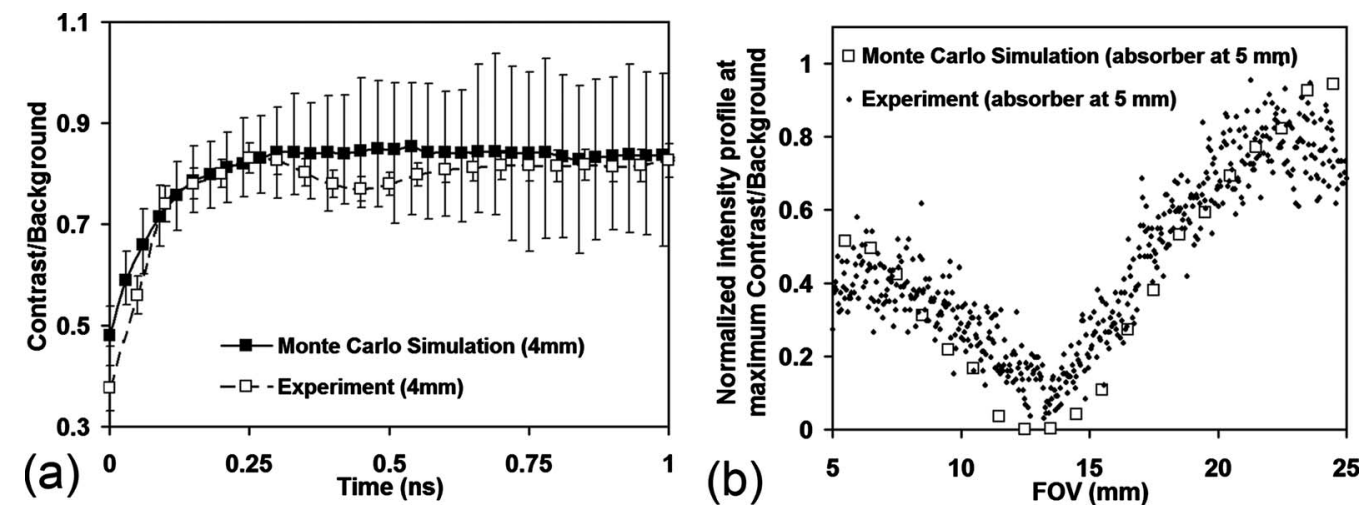

Fig. 7 (a) C/B as a function of time gate for a 4-mm-diam bile duct (simulations) and a 4-mm-diam capillary (experiments), both located at a depth of $4 \mathrm{~mm}$. (b) Comparison of diffuse reflectance profiles between simulation and experiment for a vessel depth of $5 \mathrm{~mm}$.

range of our 12-bit ICCD camera system. More specifically, no such curve could be acquired across a time-delay range of a few nanoseconds with a single camera gain setting. In Fig. 7(a), the C/B value seems relatively flat for most time delays, but this is a result of contrast and background decreasing at the same rate with time delay so that their ratio remains constant. Therefore, from an experimental point of view, there were many fewer counts at later time gates, which resulted in a "floor" effect related to the detector's limited dynamic range, and photon counts were underestimated. Therefore, the only way to acquire curves such as the one in Fig. 7(a) was to acquire data at different time-delay ranges, of a few hundred picoseconds' width at a time, with progressively increasing gain for later time gates. While acquiring data in a given time-delay range, the detector was programmed to not be active at earlier time gates so that it would not saturate. C/B for different time-delay ranges were then normalized as a fraction of the average count rate at each camera gain setting and were stitched together to form curves like the one shown in Fig. 7 (a). Though these experiments validated the MC-simulation results, they would be impractical to implement in a clinical setting due to the need for continual adjustment of gain, because later time gates have substantially fewer photons than earlier ones. A higher dynamic range system, such as the commercially available 16-bit version of the ICCD camera used in this work, could obviate the need for continual gain adjustment and therefore be more practical for use in the clinic.

Figure 7(b) shows the comparison of diffuse reflectance profiles along a line crossing through the center of the Gaussian illumination profile, across the camera FOV. The reflectance profiles were asymmetric because the illumination was incident at an angle of $30 \mathrm{deg}$ to avoid the camera being in the way of the illumination path (Fig. 2). Good agreement was observed between the MC-simulated and experimentally acquired profiles for that geometry [Fig. 7(b), time delay $350 \mathrm{ps}$. Good agreement was also observed for the maximum C/B [Fig. 8(a)] and minimum FWHM [Fig. 8(b)] values found from fitting $\mathrm{MC}$-simulated and experimental images to Eq. (1). As the profiles where asymmetric due to angled illumination, a linear correction for the slanted background was implemented before fitting to Eq. (1) for both experimental and corresponding MC data. However, the ICCD camera's limited dynamic range affected the maximum depth at which the diffuse shadow of the capillary could be detected in the reflectance profile to $\sim 7 \mathrm{~mm}$ for $\mathrm{CW}$ and to $\sim 8 \mathrm{~mm}$ for timegated illumination. Had the 16-bit version of the same commercial ICCD system been available to us, the aforementioned dynamic range problems discussed in the context of Figs. 7 and 8 would be less severe and may have enabled
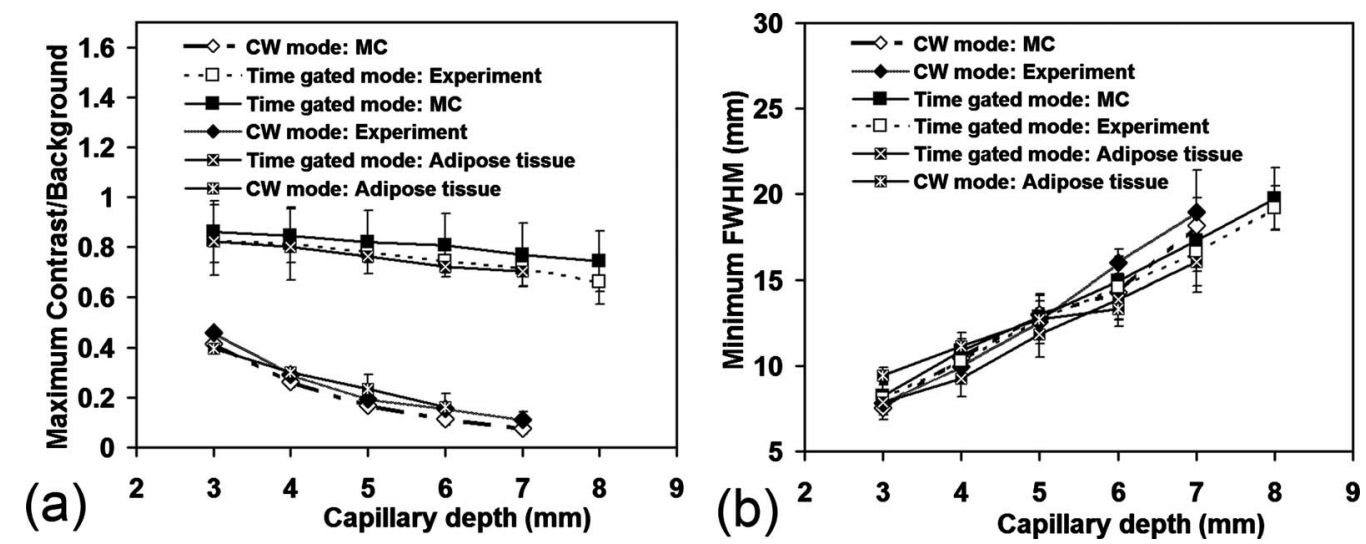

Fig. 8 Comparison between MC simulations and experiments on Intralipid and adipose tissue phantoms for (a) maximum C/B and (b) minimum FWHM values as a function of capillary depth. 
observation of the vessel at even greater depths.

In addition to the measurements on Intralipid phantoms, reflectance measurements were also performed with the capillary embedded at different depths within porcine adipose tissue. The motivation for these latter measurements was that the heterogeneous texture of adipose tissue would emulate somewhat more closely the clinical setting compared to the uniform Intralipid fluid. Reflectance measurements from the Intralipid phantom were divided with corresponding adiposeembedded capillary reflectance measurements to flatten the illumination profile. It was found that the optimal $\mathrm{C} / \mathrm{B}$ and FWHM values for adipose tissue measurements were not significantly different compared to the ones deduced from the Intralipid phantom experiments [Figs. 8(a) and 8(b)]. The only difference was that the maximum depth at which the diffuse shadow of the capillary could be detected in the reflectance profile was now reduced to $\sim 6 \mathrm{~mm}$ for $\mathrm{CW}$ and to $\sim 7 \mathrm{~mm}$ for time-gated illumination. The reduction of maximum detection depth by $\sim 1 \mathrm{~mm}$ relative to the Intralipid case was found to be due to the heterogeneity of adipose tissue, which introduced small spatial heterogeneity in the reflectance signal. Once the vessel shadow depth became comparable in magnitude to the amplitude of spatial variations in reflectance, the vessel was no longer detectable. Therefore, in the adipose tissue case, the depth detection sensitivity was limited by spatial heterogeneity of the reflectance profile in the FOV and not by the ICCD dynamic range.

\section{Discussion and Conclusions}

We have evaluated the potential use of wide-field time-gated reflectance imaging as a means to improve the visualization of tissue-embedded vessels. Though the methods presented are generalizable to any vessel type, the current work has studied how time-gated imaging at near-infrared wavelengths with an ultrafast ICCD camera can improve the detection sensitivity $(\mathrm{C} / \mathrm{B})$ and spatial resolution (FWHM) of an isolated bile duct embedded at different depths within an adipose tissue slab. MC simulations of photon propagation for broad Gaussian illumination in this tissue geometry indicated that there are optimal time gates for C/B and FWHM. More specifically, $\mathrm{C} / \mathrm{B}$ was maximum when the mean visit depth in tissue reached the vessel's center and FWHM was minimum when the mean visit depth just crossed the top of the vessel.

The salient finding of this work was that time-gated imaging attained superior $\mathrm{C} / \mathrm{B}$ and FWHM values compared to $\mathrm{CW}$ imaging for all vessel depths. Improvements in spatial resolution were modest, i.e., of the order of 15-30\% [Figs. $5(\mathrm{c})$ and $5(\mathrm{~d})]$. However, time-gated imaging could offer much higher vessel-detection sensitivity, by up to a factor of $\sim 2$, in reflectance mode [Fig. 4(c)]. Interestingly, CW imaging in transmission mode was only slightly inferior to its timegated counterpart, by $\sim 20 \%$ [Fig. 4(d)], and enabled much higher vessel-detection sensitivity than the corresponding reflectance mode [Fig. 4(b)]. Therefore, CW imaging in transmission mode, if feasible in the clinical setting, may be a good compromise between imaging system performance and cost. However, in the current implementation of imaging systems for cholecystectomy, broad Gaussian illumination is achieved by use of a lamp emitting a divergent cone of light from $\sim 1-\mathrm{m}$ distance away from the intraoperative field. ${ }^{17}$
Transillumination from the anterior side of the gallbladder would entail inserting a light source inside the patient and shining light in a manner that covers the entire intraoperative FOV from only centimeters away from the light source, which is feasible but less straightforward to implement than external illumination in reflectance geometry.

We also performed tissue-simulating phantom experiments with a time-gated ICCD camera system. Though agreement was attained with MC-simulation results (Figs. 7 and 8), performing these experiments highlighted the need for using a 16-bit camera system, or one with even higher dynamic range, for data acquisition to become practical. Furthermore, it should be acknowledged that imaging of the intraoperative FOV in a human patient would pose further practical difficulties, not encountered in tissue phantom studies. One possible challenge is that background reflectance measurements in human tissues cannot be performed and a uniformly reflecting target is used instead. ${ }^{17}$ Because the optical properties of the latter do not necessarily match the bulk human tissue optical properties, incomplete cancelation of the Lorentzian intensity profile may occur when computing the ratio of those two images. In this work, the FOV size used was substantially smaller than the FWHM of the illumination profile, which resulted in relatively flat illumination across the FOV. Under these experimental conditions, the deduced C/B and FWHM values were not sensitive to the optical properties of the tissue phantom used for background reflectance measurements, because changes in optical properties altered the reflectance near the FOV center only by a multiplicative factor. If FOV illumination can be made flat, then the primary concern that remains is that the intraoperative tissues are optically heterogeneous. ${ }^{33}$ These heterogeneities could mask a faint shadow created by a vessel in reflectance or transmission geometry and thus limit the maximum depth at which a vessel is detectable, as indicated by the adipose tissue phantom reflectance measurements performed in this work [Figs. 8(a) and $8(b)$. Though not completely representative of the clinical setting, these adipose tissue measurements indicate that vessel-detection sensitivity may be lower than what tissue phantom experiments predict and use of fluorescent contrast agents may be needed. ${ }^{34}$ Even with intrinsic contrast from the bile inside the bile duct though, the above simulations show that time-gated imaging may enhance spatial resolution and vessel-detection sensitivity relative to $\mathrm{CW}$ imaging down to a depth of several millimeters, especially in reflectance geometry. A lower cost competing technology would be CW reflectance imaging with crossed polarizers. However, this type of imaging can only enhance contrast within a few mean free paths from the tissue surface, ${ }^{35}$ which translates to $2-3 \mathrm{~mm}$ for adipose tissue optical properties at near-infrared wavelengths. Time-gated imaging shows promise to enhance vessel detection at greater depths, but a clinical evaluation of this technology is necessary to decide whether its benefits outweigh its higher cost.

\section{References}

1. C. G. Hoelen, F. F. de Mul, R. Pongers, and A. Dekker, "Threedimensional photoacoustic imaging of blood vessels in tissue," Opt. Lett. 23(8), 648-650 (1998).

2. R. J. Zemp, R. Bitton, M. L. Li, K. K. Shung, G. Stoica, and L. V. Wang, "Photoacoustic imaging of the microvasculature with a high- 
frequency ultrasound array transducer," J. Biomed. Opt. 12(1), 010501 (2007).

3. B. Li, B. Majaron, J. A. Viator, T. E. Milner, Z. Chen, Y. Zhao, H. Ren, and J. S. Nelson, "Accurate measurement of blood vessel depth in port wine stained human skin in vivo using pulsed photothermal radiometry," J. Biomed. Opt. 9(5), 961-966 (2004).

4. J. G. Fujimoto, "Optical coherence tomography for ultrahigh resolution in vivo imaging," Nat. Biotechnol. 21(11), 1361-1367 (2003).

5. A. Corlu, R. Choe, T. Durduran, K. Lee, M. Schweiger, S. R. Arridge, E. M. C. Hillman, and A. G. Yodh, "Diffuse optical tomography with spectral constraints and wavelength optimization," Appl. Opt. 44(11), 2082-2093 (2005).

6. S. Srinivasan, B. W. Pogue, S. D. Jiang, H. Dehghani, and K. D. Paulsen, "Spectrally constrained chromophore and scattering nearinfrared tomography provides quantitative and robust reconstruction," Appl. Opt. 44(10), 1858-1869 (2005).

7. G. Boverman, Q. Fang, S. A. Carp, E. L. Miller, D. H. Brooks, J. Selb, R. H. Moore, D. B. Kopans, and D. A. Boas, "Spatio-temporal imaging of the hemoglobin in the compressed breast with diffuse optical tomography," Phys. Med. Biol. 52(12), 3619-3641 (2007).

8. S. R. Arridge, "Optical tomography in medical imaging," Inverse Probl. 15(2), R41-R93 (1999).

9. B. Brooksby, S. Srinivasan, S. D. Jiang, H. Dehghani, B. W. Pogue, K. D. Paulsen, J. Weaver, C. Kogel, and S. P. Poplack, "Spectral priors improve near-infrared diffuse tomography more than spatial priors," Opt. Lett. 30(15), 1968-1970 (2005).

10. V. E. Pera, E. L. Heffer, H. Siebold, O. Schutz, S. HeywangKobrunner, L. Gotz, A. Heinig, and S. Fantini, "Spatial secondderivative image processing: an application to optical mammography to enhance the detection of breast tumors," J. Biomed. Opt. 8(3), 517-524 (2003).

11. D. S. Kepshire, S. C. Davis, H. Dehghani, K. D. Paulsen, and B. W. Pogue, "Subsurface diffuse optical tomography can localize absorber and fluorescent objects but recovered image sensitivity is nonlinear with depth," Appl. Opt. 46(10), 1669-1678 (2007).

12. N. Liu, A. Sassaroli, and S. Fantini, "Paired-wavelength spectral approach to measuring the relative concentrations of two localized chromophores in turbid media: an experimental study," J. Biomed. Opt. 12(5), 051602 (2007)

13. A. V. Bykov, A. V. Priezzhiev, and R. Myllyla, "Spatial resolved diffuse reflection as a tool for determination of size and embedding depth of blood vessels," in Proc. SPIE 6629, 66291P (2007).

14. S. Kukreti, A. Cerussi, B. Tromberg, and E. Gratton, "Intrinsic tumor biomarkers revealed by novel double-differential spectroscopic analysis of near-infrared spectra," J. Biomed. Opt. 12(2), 020509 (2007).

15. I. Nishidate, T. Maeda, Y. Aizu, and K. Niizeki, "Visualizing depth and thickness of a local blood region in skin tissue using diffuse reflectance images," J. Biomed. Opt. 12(5), 054006 (2007).

16. D. Piao, H. Xie, W. L. Zhang, J. S. Krasinski, G. L. Zhang, H. Dehghani, and B. W. Pogue, "Endoscopic, rapid near-infrared optical tomography," Opt. Lett. 31(19), 2876-2878 (2006).

17. K. J. Zuzak, S. C. Naik, G. Alexandrakis, D. Hawkins, K. Behbehani, and E. H. Livingston, "Characterization of a near-infrared laparoscopic hyperspectral imaging system for minimally invasive surgery," Anal. Chem. 79(12), 4709-4715 (2007).

18. E. H. Livingston, J. A. Miller, B. Coan, and R. V. Rege, "Costs and utilization of intraoperative cholangiography," J. Gastrointest Surg. 11(9), 1162-1167 (2007).

19. D. A. Osborne, G. Alexander, B. Boe, and E. E. Zervos, "Laparoscopic cholecystectomy: past, present, and future," Surg. Technol. Int. 15, 81-85 (2006).
20. R. Orlando, 3rd, J. C. Russell, J. Lynch, and A. Mattie, "Laparoscopic cholecystectomy: a statewide experience. The Connecticut Laparoscopic Cholecystectomy Registry," Arch. Surg. 128(5), 494498 (1993); discussion 128(5), 498-499 (1993).

21. J. F. Buell, D. C. Cronin, B. Funaki, A. Koffron, A. Yoshida, A. Lo, J. Leef, and J. M. Millis, "Devastating and fatal complications associated with combined vascular and bile duct injuries during cholecystectomy," Arch. Surg. 137(6), 703-708 (2002); discussion 137(6), 708-710 (2002).

22. D. J. Hall, J. C. Hebden, and D. T. Delpy, "Imaging very-lowcontrast objects in breastlike scattering media with a time-resolved method," Appl. Opt. 36(28), 7270-7276 (1997).

23. D. J. Hall, J. C. Hebden, and D. T. Delpy, "Evaluation of spatial resolution as a function of thickness for time-resolved optical imaging of highly scattering media," Med. Phys. 24(3), 361-368 (1997).

24. K. Michielsen, H. DeRaedt, and N. Garcia, "Time-gated transillumination and reflection by biological tissues and tissuelike phantoms: simulation versus experiment," J. Opt. Soc. Am. A 14(8), 1867-1871 (1997).

25. D. A. Boas, J. P. Culver, J. J. Stott, and A. K. Dunn, "Three dimensional Monte Carlo code for photon migration through complex heterogeneous media including the adult human head," Opt. Express 10(3), 159-170 (2002).

26. A. M. K. Enejder, J. Swartling, P. Aruna, and S. Andersson-Engels, "Influence of cell shape and aggregate formation on the optical properties of flowing whole blood," Appl. Opt. 42(7), 1384-1394 (2003).

27. M. J. Holboke, B. J. Tromberg, X. Li, N. Shah, J. Fishkin, D. Kidney, J. Butler, B. Chance, and A. G. Yodh, "Three-dimensional diffuse optical mammography with ultrasound localization in a human subject," J. Biomed. Opt. 5(2), 237-247 (2000).

28. A. Kienle, L. Lilge, M. S. Patterson, R. Hibst, R. Steiner, and B. C. Wilson, "Spatially resolved absolute diffuse reflectance measurements for noninvasive determination of the optical scattering and absorption coefficients of biological tissue," Appl. Opt. 35(13), 23042314 (1996).

29. D. J. Maitland, J. T. Walsh, and J. B. Prystowsky, "Optical-properties of human gallbladder tissue and bile," Appl. Opt. 32(4), 586-591 (1993).

30. G. Mitic, J. Kolzer, J. Otto, E. Plies, G. Solkner, and W. Zinth, "Time-gated transillumination of biological tissues and tissuelike phantoms," Appl. Opt. 33(28), 6699-6710 (1994).

31. S. Srinivasan, B. W. Pogue, S. D. Jiang, H. Dehghani, C. Kogel, S. Soho, J. J. Gibson, T. D. Tosteson, S. P. Poplack, and K. D. Paulsen, "Interpreting hemoglobin and water concentration, oxygen saturation, and scattering measured in vivo by near-infrared breast tomography," Proc. Natl. Acad. Sci. U.S.A. 100(21), 12349-12354 (2003).

32. J. F. Beek, P. Blokland, P. Posthumus, M. Aalders, J. W. Pickering, H. J. Sterenborg, and M. J. van Gemert, "In vitro double-integratingsphere optical properties of tissues between 630 and 1064 nm," Phys. Med. Biol. 42(11), 2255-2261 (1997).

33. K. J. Zuzak, S. C. Naik, G. Alexandrakis, D. Hawkins, K. Behbehani, and E. Livingston, "Intraoperative bile duct visualization using nearinfrared hyperspectral video imaging," Am. J. Surg. 195(4), 491-497 (2008).

34. D. J. Hall, U. Sunar, S. Farshchi-Heydari, and S. H. Han, "In vivo simultaneous monitoring of two fluorophores with lifetime contrast using a full-field time domain system," Appl. Opt. 48(10), D74-D78 (2009).

35. D. A. Zimnyakov, J. T. Oh, Y. P. Sinichkin, V. A. Trifonov, and E. V. Gurianov, "Polarization reflectance spectroscopy of biological tissues: diagnostic applications," J. Opt. Soc. Am. A 21(1), 59-70 (2004). 